\title{
Methanol formation: A Monte Carlo study
}

\author{
Ankan Das $^{1}$, Kinsuk Acharyya ${ }^{2}$, Sonali Chakrabarti ${ }^{3,1}$, and \\ Sandip K. Chakrabarti ${ }^{2,1}$ \\ ${ }^{1}$ Indian Centre for Space Physics, \\ Chalantika 43, Garia Station Road, Kolkata 700084, India \\ email: ankan@csp.res.in \\ ${ }^{2}$ S. N. Bose National Centre for Basic Sciences \\ Salt Lake, Kolkata 700098, India \\ email: acharyya@bose.res.in, chakraba@bose.res.in \\ ${ }^{3}$ Maharaja Manindra Chandra College \\ 20 Ramakanta Bose Lane, Kolkata 700003, India \\ email: sonali@csp.res.in
}

\begin{abstract}
We carry out a Monte-Carlo simulation to study the formation of methanol on the grain surfaces. We found that the recombination efficiencies are strongly dependent on the extrinsic properties of the grain, such as the number of sites on the grain surface and the flux of the accreting matter. This uses the concept of effective grain surface (denoted through a factor $\alpha$ ) area which changes as the grain is populated.
\end{abstract}

Keywords. ISM: abundances, ISM: molecules, methods: numerical, molecular processes

\section{Introduction}

It is now well known that the grain chemistry plays a vital role in the chemical evolutions of the molecular cloud. The rate equation method is very extensively used by several authors to study the grain surface chemistry (Hasegawa et al. 1992, Roberts \& Herbst 2002, Acharyya et al. 2005). However, this method is only applicable when there are large number of reactants on the grain surface. Given the fact that the interstellar medium is very dilute, very often the criteria for using the rate equation are not fulfilled. Furthermore, it is assumed that the recombination efficiency is independent of the surface area of the grain. The only advantage of this method is that computationally it is faster and it can very easily be coupled with the gas phase reactions. More realistic method to handle the grain chemistry is the Monte-Carlo approach. This is very accurate method because here we can trace each and every species over the entire period of time. The major disadvantage of this is that it is computationally time consuming. Using MonteCarlo approach Chakrabarti et al. $(2006 \mathrm{a}, \mathrm{b})$ introduced a new concept of the effective grain surface area and showed that it is this effective area which must be used for the grain chemistry. It was argued that the surface area $S$ in the usual rate equation is to be replaced by $S^{\alpha}$ where $\alpha$ is to be determined from Monte-Carlo method for a few cases and extrapolated value is to be used in the equation. Its usefulness was demostrated while computing the formation of $\mathrm{H}_{2}$ molecules on the grains. In the present work, we carry out a similar analysis to study the formation of water and methanol on the grains.

\section{Procedure}

We study the chemical evolution on grains assuming $\mathrm{H}, \mathrm{O}$ and $\mathrm{CO}$ as the sole accreting species. For the sake of simplicity, we assume that a grain surface is a square lattice $S$ number of sites. We assume further that each site has four nearest neighbours, as in an fcc[100] lattice. In order to mimic the spherical grain structure, we assume a periodic 

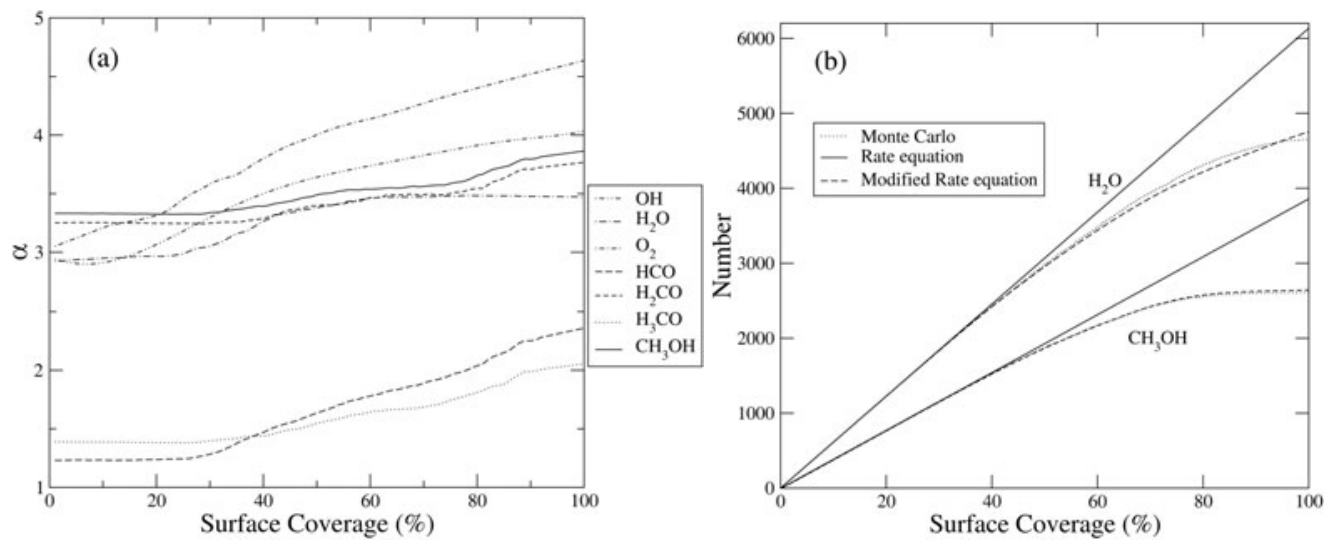

Figure 1. In (a) we plot $\alpha$ for various species. In (b) we compare our results with the modified rate equation method, where we replaced $S$ by $S^{\alpha}$ and with the original rate equation method.

boundary condition. The four major steps to handle the grain chemistry are the accretion, diffusion, reaction and evaporation. We execute every step by the appropriate use of the random number generator. Keeping in mind that there are two types of reaction schemes (Eley-Rideal and Langmuir-Hinselwood), we continue our simulations for different models assuming different procedure for the accretion of the gas phase species.

\section{Results}

In Figure 1a, we plot $\alpha$ for various species as a function of the surface coverage for an Olivine grain at $10 \mathrm{~K}$ having $10^{4}$ sites. We note that $\alpha$ is not unity for any of these species. Up to about $30 \%$ of the surface coverage $\alpha$ remains roughly constant, but after that they rise as the surface gets filled up. In Figure 1b, we made a comparison of the abundances of two of the most important species, namely, $\mathrm{H}_{2} \mathrm{O}$ and $\mathrm{CH}_{3} \mathrm{OH}$ in different methods. The results of our simulation clearly agree with those from the modified rate equation, but not with those from the original rate equation. This indicates that the $\alpha \mathrm{s}$ we introduced are very important and their effects are to be taken into account for an accurate estimation of the species from the grain chemistry.

\section{Concluding Remarks}

According to the observations, the solid state methanol abundance should be $5 \%$ to $30 \%$ (Gibb et al. 2000) with respect to $\mathrm{H}_{2} \mathrm{O}$. We carried out our simulation for the low gas phase abundance (Case I: density of $\mathrm{H}_{2} 10^{3} \mathrm{~cm}^{-3}$ ) and for the high gas phase abundance (Case II: density of $\mathrm{H}_{2} 10^{5} \mathrm{~cm}^{-3}$ ). We found that for different models this number varies from $(\sim 43 \%-\sim 57 \%)$ for Case I and from $(4 \%-6 \%)$ for Case II. Thus the observed abundance must be from some intermediate region of the cloud. One of our important findings is that the parameter $\alpha$ strongly depends on the surface population of the grain surface. This deviates significantly from unity, the usual value in existing rate equation.

\section{References}

Acharyya, K., Chakrabarti, S. K., \& Chakrabarti, S. 2005, MNRAS, 361, 550

Chakrabarti, S. K., Das, A., Acharyya, K., \& Chakrabarti, S. 2006a, A\&A, 457, 167

Chakrabarti, S. K., Das, A., Acharyya, K., \& Chakrabarti, S. 2006b, BASI, 34, 299

Gibb, E. L., et al. 2000, ApJ, 536, 347

Hasegawa, T., Herbst, E., \& Leung, C. M. 1992, ApJS, 82, 167

Roberts, H. \& Herbst, E. 2002, A\& A, 395, 233 